\title{
THE STIELTJES STRING AND ITS ASSOCIATED NODAL POINTS
}

\author{
Che-Wei Tsao And Chun-Kong LAW
}

Abstract. Based on the theory of Stieltjes strings first introduced by Gantmakher and Krein in [4], we define the nodal points for a Stieltjes string. We show that when the eigenvalue is maximal, there are exactly $n+1$ nodal points for the D-D problem and $n$ nodal points for the $\mathrm{D}-\mathrm{N}$ problem, where $n$ is the total number of non-zero point masses. We also find the position of these nodal points in terms of continued fractions involving the point masses $m_{1}, \ldots, m_{j}$ and lengths $l_{0}, \ldots, l_{j-1}$ in between the positions of these masses.

Mathematics subject classification (2010): 34C10, 39A12, 47B36.

Keywords and phrases: Stieltjes strings, characteristic functions, continued fractions.

\section{REFERENCES}

[1] O. BOY KO AND V. PIVOVARCHIK, The inverse three-spectral problem for a Stieltjes string and the inverse problem with one-dimensional damping, Inverse Problems 24 (2008) no.1, 015019, 13 pp.

[2] O. BOYKO AND V. PIVOVARCHIK, Inverse spectral problem for a star graph of Stieltjes strings, Methods Funct. Anal. Topology 14 (2008) no.2, 159-167.

[3] CHU, M. T, Inverse eigenvalue problems, SIAM Review, 40 (1998) no.1, 1-39.

[4] F. P. Gantmakher And M. G. Krein, Oscillating Matrices and Kernels and Small Vibrations of Mechanical Systems, Amer. Math. Soc., Providence, 2002.

[5] R. A. Horn And C. R. Johnson, Matrix Analysis, Cambridge University Press, 1990.

[6] I. S. KaC AND M. G. KRein, On the spectral function of the string, Amer. Math. Soc. Translations, Ser.2, 103 (1974) 19-102.

[7] C. K. LAW, V. PIVovarChiK AND W. C. WANG, A polynomial identity and its application to inverse spectral problems in Stieltjes Strings, Operators and Matrices, 7 (2013) no.3, 603-617.

[8] O. Martynuk, V. Pivovarchik, C. TRetTer, Inverse problem for a damped Stieltjes string from parts of spectra, Appl. Anal., 94 (2015) no.12, 2605-2619.

[9] M. MöLler, V. PivovarchiK, Damped star graphs of Stieltjes strings, Proc. Amer. Math. Soc., 145 (2017) no.4, 1717-1728.

[10] V. PIVOVARCHIK, Existence of a tree of Stieltjes strings corresponding to two given spectra, J. Phys. A, 42 (2009) no.37, 375213, 16 pp.

[11] G. TeSCHL, Jacobi Operators and Completely Integrable Nonlinear Lattices, Math. Surv. Mono. 72, Amer. Math. Soc., Rhode Island, 2000. 